\title{
Mössbauer Effect Probe of Local Jahn-Teller distortion in Fe-doped Colossal Magnetoresistive Manganites
}

\author{
Zhao-hua Cheng*, Zhi-hong Wang, Nai-li Di, Zhi-qi Kou, Guang-jun Wang, Rui-wei Li, Yi \\ Lu, Qing-an Li, and Bao-gen Shen \\ State Key Laboratory of Magnetism and International Center for Quantum \\ Structures, Institute of Physics, Chinese Academy of Sciences, Beijing \\ 100080, P.R. China \\ R.A. Dunlap \\ Department of Physics, Dalhousie University, Halifax, NovaScotia,Canada B3H \\ $3 J 5$
}

\begin{abstract}
Local structure of the Fe-doped $\mathrm{La}_{1-x} \mathrm{Ca}_{x} \mathrm{MnO}_{3}(\mathrm{x}=0.00-1.00)$ compounds has been investigated by means of Mössbauer spectroscopy. ${ }^{57} \mathrm{Fe}$ Mössbauer spectra provide a direct evidence of Jahn-Teller distortion in these manganites. On the basis of Mössbauer results, the Jahn-Teller coupling was estimated. It is noteworthy that Ca-concentration dependence of Jahn-Teller coupling strength is very consistent with the magnetic phase diagram. Our results reveal that Mössbauer spectroscopy can not only detect the local structural distortion, but also provide a technique to investigate Jahn-Teller coupling of Fe-doped $\mathrm{La}_{1-x} \mathrm{Ca}_{x} \mathrm{MnO}_{3}$ colossal magnetoresistive perovskites.

PACS: 61.10.-i; 76.80.+y; 75.30. Vn

*Corresponding author
\end{abstract}

Typeset using REVTEX 
The discovery of colossal magnetoresistance (CMR) in the manganese perovskites $\mathrm{La}_{1-x} \mathrm{Ca}_{x} \mathrm{MnO}_{3}$ has recently attracted significant scientific attention. ${ }^{1,2}$ More and more evidence has indicated that the double-exchange coupling model alone is not sufficient to explain the CMR effect, and strong electron-lattice coupling plays an important role in determining the observed resistivity behavior and the magnetic transition temperature. ${ }^{3-7}$ Millis has argued that there are two types of lattice effects in $\mathrm{ABO}_{3}$-type magnetoresistive manganese perovskites. ${ }^{7}$ One is the tolerance factor, $t=\frac{R_{A}+R_{O}}{\sqrt{2}\left(R_{B}+R_{O}\right)}$, where $R_{A}, R_{B}$ and $R_{O}$ are the radii of $\mathrm{A}, \mathrm{B}$ and $\mathrm{O}$ ions, respectively. ${ }^{4}$ The other one is the local structural distortion of $\mathrm{MnO}_{6}$ octahedron resulting from the $\mathrm{Mn}^{3+}$ Jahn-Teller effect. Since the longrange structural distortion decreases rapidly in $\mathrm{La}_{1-x} \mathrm{Ca}_{x} \mathrm{MnO}_{3}$ compounds with increasing Ca concentration, ${ }^{8}$ a local structural probe, such as Mössbauer spectroscopy is required to analyze the distortion of the $\mathrm{MnO}_{6}$ octahedron.

The idea of using Mössbauer spectroscopy to detect local structure is based on the quadrupole splitting $(\Delta)$ that is very sensitive to the distribution of surrounding electrons of resonant nuclei. A few percent of Fe substitution for $\mathrm{Mn}$ in $\mathrm{La}_{1-x} \mathrm{Ca}_{x} \mathrm{MnO}_{3}$ compounds can be used as a micro-probe to detect the symmetry of the nearest-neighbor $\mathrm{O}^{2-}$ ions in the $\mathrm{Mn}(\mathrm{Fe}) \mathrm{O}_{6}$ octahedron. Furthermore, early studies have shown that $\mathrm{Mn}^{3+}$ ions are mainly replaced by $\mathrm{Fe}^{3+}$ ions in this Fe-doping range, ${ }^{9}$ and that both ions have identical ionic radii $(0.645 \AA)$ in six-fold octahedral coordination. ${ }^{10}$ Therefore, the substitution of $\mathrm{Fe}^{3+}$ for $\mathrm{Mn}^{3+}$ does not change the tolerance factor, and consequently, the Jahn-Teller effect can be investigated separately.

Several papers have been published using Mössbauer spectroscopy, ${ }^{11-14}$ but the local structural distortion of $\mathrm{Mn}(\mathrm{Fe}) \mathrm{O}_{6}$ octahedra and Jahn-Teller effect were not yet investigated by this technique. In this Letter, we focused our investigation on the local structural information regarding $\mathrm{Mn}(\mathrm{Fe}) \mathrm{O}_{6}$ octahedra. We present ${ }^{57} \mathrm{Fe}$ Mössbauer spectra recorded by a Wissel System constant acceleration Mössbauer spectrometer with a ${ }^{57} \mathrm{Co}(\mathrm{Pd})$ source that not only clearly indicate the presence of quadrupole splitting but also show the occurrence of a second quadrupole split doublet. As an example, Mössbauer spectrum of 
$\mathrm{La}_{0.69} \mathrm{Ca}_{0.31} \mathrm{Mn}_{0.91} \mathrm{Fe}_{0.09} \mathrm{O}_{3}$ polycrystalline powders prepared by conventional solid-state reaction method illustrated in Fig. 1(a) can be best fitted with two doublets. The more intense doublet $(\simeq 90 \%)$ has a center shift $\delta=0.339 \pm 0.010 \mathrm{~mm} / \mathrm{s}$ relative to $\alpha$-Fe at room temperature, and a quadrupole splitting $\Delta=0.235 \pm 0.021 \mathrm{~mm} / \mathrm{s}$, which are quite similar with previous reports. ${ }^{11-14}$ This center shift is typical value of high-spin $\mathrm{Fe}^{3+}$ with octahedral coordination. The weaker doublet $(\simeq 10 \%)$ has $\delta=0.147 \pm 0.043 \mathrm{~mm} / \mathrm{s}$ and $\Delta=$ $0.672 \pm 0.085 \mathrm{~mm} / \mathrm{s}$. This center shift is in good agreement with that of low-spin $\mathrm{Fe}^{4+}$ in $\mathrm{SrFeO}_{3}$ compound ${ }^{15}$ The quadrupole splitting for both $\mathrm{Fe}^{3+}$ and $\mathrm{Fe}^{4+}$ ions confirms the local distortion of the $\mathrm{Mn}(\mathrm{Fe}) \mathrm{O}_{6}$ octahedron.

Since too much $\mathrm{Fe}$ as a dopant alters the electronic and magnetic properties significantly, ${ }^{12,16}$ we selected some typical samples $(\mathrm{x}=0.31,0.50$ and 0.60$)$ containing smaller concentration of $\mathrm{Fe}(4 \mathrm{at} . \%)$ to reduce the Fe-O-Fe contact and carried out x-ray diffraction (XRD) and Mössbauer measurements again. XRD pattern indicated that there is no change in structure and lattice parameters between samples with different Fe concentrations due to the same radii of $\mathrm{Mn}^{3+}$ and $\mathrm{Fe}^{3+}$ ions. Mössbauer spectra of samples with lower $\mathrm{Fe}$ concentration demonstrate that the center shift $\delta$, quadrupole splitting $\Delta$ and line width corresponding to $\mathrm{Fe}^{3+}$ ions are in good agreement with that of $\mathrm{Fe}^{3+}$ ions in the samples with 9at.\% Fe concentrations. These measurements confirm that Fe-O-Fe contacts have no obvious contribution to Mössbauer parameters at temperatures $\mathrm{T}>\mathrm{T}_{C}$. The doublet originated from low-spin $\mathrm{Fe}^{4+}$ ion was not detected due to the lower Fe substitution. Mössbauer spectra of the samples containing lower Fe concentration also indicate a local distortion of the $\mathrm{Mn}(\mathrm{Fe}) \mathrm{O}_{6}$ octahedron in La-Ca-Mn-O perovskites. The fact that the Jahn-Teller distortion in the perovskites with $\mathrm{x}>0.2$ is detected by Mössbauer effect, rather than by XRD technique implies that this distortion is a dynamic one, rather than a static one since the time scale probed by Mössbauer effect is much smaller than that of XRD technique. One possible reason for the different information obtained by these two techniques is due to their different time scales.

The local structural distortion of $\mathrm{MnO}_{6}$ octahedra resulting from the Jahn-Teller effect 
of high-spin $\mathrm{Mn}^{3+}$ ions removes the degeneracy of the $e_{g}$ and $t_{2 g}$ orbitals so as to make some energy levels more stable. The $e_{g}$ orbital group is separated into two energy levels, $d_{z^{2}}$ and $d_{x^{2}-y^{2}}$. The $t_{2 g}$ orbital group is split into a non-degenerate energy level and a two-fold degenerate energy level in the trigonally or tetragonally distorted octahedra. The two-fold degenerate energy level will further split in highly distorted octahedra. The energy separation of the upper-level orbitals has been shown to be larger than that of the lower-level orbitals. Since the Jahn-Teller distortion strongly influences the electron hopping process between the upper-level orbitals of $\mathrm{Mn}^{3+}$ and $\mathrm{Mn}^{4+}$ ions in $\mathrm{La}_{1-x} \mathrm{Ca}_{x} \mathrm{MnO}_{3}$ perovskites, and consequently determines the ferromagnetic and electrical behaviors, we only need to consider the upper-level splitting of $e_{g}$ orbitals. The energy separation between $d_{z^{2}}$ and $d_{x^{2}-y^{2}}, E_{J T}$, can be described as ${ }^{17}$

$$
\begin{aligned}
& E_{J T}=4 D_{s}+5 D_{t} \\
& D_{s}=\frac{e}{14} \sqrt{\frac{5}{\pi}} A_{20}<r^{2}>_{3 d} \\
& D_{t}=\frac{e}{14 \sqrt{\pi}} T<r^{4}>_{3 d}
\end{aligned}
$$

where $A_{20}=\sqrt{\frac{\pi}{5}} \sum \frac{q_{i}\left(3 \cos ^{2} \theta_{i}-1\right)}{R_{i}^{3}}$ is the second-order crystal-electric-field (CEF) coefficient. The electric charge, $q_{i}$, of the $\mathrm{i}^{\text {th }}$ surrounding ion should be effective charge as determined by a consideration of the shielding and polarization of inner-shell electrons of the central ion. $R_{i}$ is the radial distance of the ith surrounding ion from the central ion, and $\theta_{i}$ is its polar angle in spherical coordinates. $A_{20}$ is zero for cubic or perfectly octahedral symmetry. $T=A_{40}\left(o c t^{\prime}\right)-A_{40}(o c t)$ is the difference between the fourth-order CEF coefficient of a distorted $\mathrm{MnO}_{6}$ octahedron, $A_{40}\left(o c t^{\prime}\right)$, and that for perfect octahedral symmetry, $A_{40}($ oct $)$, where $A_{40}=\frac{\sqrt{\pi}}{4} \sum \frac{q_{i}}{R_{i}^{5}}\left(\frac{35}{3} \cos ^{4} \theta_{i}-10 \cos ^{2} \theta_{i}+1\right)$. Finally, $\left\langle r^{n}>_{3 d}\right.$ is the expectation value of the $\mathrm{n}^{\text {th }}$ power, $r^{n}$, of the radial distance of a $3 d$ orbital from the nucleus.

Using the Mn-O bond lengths and the expectation values of $\left\langle r^{2}>_{3 d}\right.$ and $<r^{4}>_{3 d}$ for $\mathrm{Mn}^{3+}$ ions ${ }^{18}$ it can easily be shown from eqs. (2) and (3) that $D_{t}<<D_{s}$. Therefore, the energy separation between and orbitals arises mainly from the contribution of the second-order CEF coefficient $A_{20}$ of the distorted octahedra and eq. (1) can be written as: 


$$
E_{J T} \simeq 4 D s=\frac{2 e}{7} \sqrt{\frac{5}{\pi}} A_{20}<r^{2}>_{3 d}
$$

Although the relationship between $E_{J T}$ and $A_{20}$ is relatively simple, small errors in X-ray structural data can cause very large errors in the values of $A_{20}$. Moreover, since the surrounding ionic charge is not concentrated exclusively at the lattice positions as assumed in the point charge model, the contribution of inner-shell electrons' shielding and the polarization of the central ions should be taken into account as well. Therefore, it is difficult to obtain a reliable result on the basis of x-ray diffraction data alone. However, Mössbauer spectroscopy can be employed to determine the value of $A_{20}$ by a measurement of the quadrupole splitting as described below.

In the case of ${ }^{57} \mathrm{Fe}$ Mössbauer effect, the quadrupole splitting, $\Delta$, can be written as ${ }^{19}$

$$
\Delta=\frac{e Q V_{z z}}{2}\left(1+\frac{\eta^{2}}{3}\right)^{1 / 2}
$$

where $Q$ is the electric quadrupole moment of the ${ }^{57} \mathrm{Fe}$ nucleus, $\eta$ is the asymmetry parameter and $V_{z z}$ is the principal component of the electric field gradient (EFG) at the nucleus. This final quantity includes contributions from valence electrons of both the Fe ion $V_{Z Z}(\mathrm{Fe})$, and the surrounding ions, $V_{Z Z}$ (latt). This may be written as

$V_{Z Z}(t o t)=V_{Z Z}(F e)+V_{Z Z}(l a t t)$

where

$$
\begin{aligned}
& V_{Z Z}(\text { latt })=\sum \frac{q_{i}\left(3 \cos ^{2} \theta_{i}-1\right)}{R_{i}^{3}}=\sqrt{\frac{5}{\pi}} A_{20} \\
& V_{Z Z}(F e)=\sum P_{s} V_{z z}(s)
\end{aligned}
$$

where $P_{s}$ is the probability of the valence electrons at different orbitals. The values of $V_{z z}(\mathrm{~s})$ for $d_{x^{2}-y^{2}}, d_{z^{2}}, d_{x y}, d_{y z}$ and $d_{z x}$ orbitals are $+\frac{4 e}{7}<r^{-3}>,-\frac{4 e}{7}<r^{-3}>,+\frac{4 e}{7}<r^{-3}>$, $-\frac{2 e}{7}<r^{-3}>,-\frac{2 e}{7}<r^{-3}>$, respectively. ${ }^{19}$ Thus, the contribution from the valence electrons of the Fe cations to the EFG at the nucleus depends on their configuration, i.e. the valencestate and spin-state. In the case of perfect octahedra, the four valence electrons of a lowspin $\mathrm{Fe}^{4+}$ ion occupy the three-fold degenerate $t_{2 g}$ orbitals $\left(d_{x y}, d_{y z}\right.$ and $\left.d_{z x}\right)$ with equal probability in the ground state and produce no $\mathrm{EFG}, V_{z z}(\mathrm{Fe})=0$. However, in the case of lower symmetry, the degeneracy is removed and electrons will preferentially occupy the 
low-lying energy level and a non-zero EFG will be produced in an $\mathrm{Fe}^{4+}$ ion by its valence electrons, $V_{z z}(\mathrm{Fe}) \neq 0$. For high-spin $\mathrm{Fe}^{3+}$, however, five valence electrons each occupy one of the five $3 d$ orbitals. The half-filled shell is spherically symmetric regardless of the distribution of surrounding ions and the valence electrons make no contribution to the $\mathrm{EFG}, V_{z z}(\mathrm{Fe})=0$. Therefore, the valence electrons will make no contribution to the quadrupole splitting for either $\mathrm{Fe}^{3+}$ or $\mathrm{Fe}^{4+}$ ions in perfect octahedral symmetry, but will yield a contribution to the quadrupole splitting for $\mathrm{Fe}^{4+}$ in distorted octahedra. Compared with $\mathrm{Fe}^{3+}$ ion, about $0.4 \mathrm{~mm} / \mathrm{s}$ larger in quadrupole splitting corresponding to $\mathrm{Fe}^{4+}$ ions may be related to valence electron contribution.

Since $\Delta$ at the nucleus of $\mathrm{Fe}^{3+}$ ion is solely the result of contributions from the surrounding ions, the values of $A_{20}$ can be obtained from

$$
A_{20}=\sqrt{\frac{4 \pi}{5}} \frac{\Delta\left(F e^{3+}\right)}{e Q\left(1+\frac{\eta 2}{3}\right)^{1 / 2}}
$$

Therefore, the relationship between $E_{J T}$ and the quadrupole splitting at $\mathrm{Fe}^{3+}$ ion $\Delta\left(\mathrm{Fe}^{3+}\right)$ can be written as

$$
E_{J T}=\frac{4 \Delta\left(F e^{3+}\right)}{7 Q\left(1+\frac{\eta^{2}}{3}\right)^{1 / 2}}<r^{2}>_{3 d}
$$

where $\eta=0$ for axial symmetry. The quadrupole splitting for Mössbauer experiment is generally in unit of $\mathrm{mm} / \mathrm{s}$. Here it should be converted to $\mathrm{eV}$ by a factor of $E \gamma / c$, where $E \gamma=14.4 \mathrm{keV}$ for $I=3 / 2 \rightarrow 1 / 2$ transition of ${ }^{57} \mathrm{Fe}$ and $c=3 \times 10^{11} \mathrm{~mm} / \mathrm{s}$ is the velocity of light.

Using a approximation of the expectation value $<r^{2}>$ of free $\mathrm{Mn}^{3+}$ ions, $<r^{2}>=0.3535$ $\AA^{2}$ and $Q=0.28 \times 10^{-24} \mathrm{~cm}^{2},{ }^{18,20}$ the Jahn-Teller coupling, $E_{J T}$, in $\mathrm{La}_{1-x} \mathrm{Ca}_{x}(\mathrm{Mn}, \mathrm{Fe}) \mathrm{O}_{3}$ compounds can be estimated. Fig. 2 illustrates the quadrupole splitting corresponding to $\mathrm{Fe}^{3+}$ ions and Jahn-Teller coupling in $\mathrm{La}_{1-x} \mathrm{Ca}_{x} \mathrm{Mn}_{0.91} \mathrm{Fe}_{0.09} \mathrm{O}_{3}(\mathrm{x}=0.00-1.00)$ and $\mathrm{La}_{1-x} \mathrm{Ca}_{x} \mathrm{Mn}_{0.96} \mathrm{Fe}_{0.04} \mathrm{O}_{3}$ ( $\left.\mathrm{x}=0.31-0.60\right)$ as a function of Ca concentration. It is noteworthy that the Ca-concentration dependence of the Jahn-Teller coupling strength is consistent well with the magnetic phase diagram, implying a direct relationship between Jahn-Teller effect as well as electric and magnetic properties in these perovskites. The Jahn-Teller coupling is indeed strong in $\mathrm{LaMn}_{0.91} \mathrm{Fe}_{0.09} \mathrm{O}_{3}$. With increasing Ca concentration, the energy sepa- 
ration between $d_{z^{2}}$ and $d_{x^{2}-y^{2}}$ decreases from about $1.4 \mathrm{eV}$ for $\mathrm{x}=0$ to about $0.83 \mathrm{eV}$ for $\mathrm{x}=0.27-0.39$. The transport properties of these perovskites are governed by the interplay of the double-exchange coupling between $\mathrm{Mn}^{3+}$ and $\mathrm{Mn}^{4+}$ ions as well as the Jahn-Teller coupling. For the samples with $\mathrm{x}=0.27-0.39$, the relatively weak Jahn-Teller coupling delocalizes the electrons and makes the hoping process easier via double-exchange coupling. Therefore, these samples show a ferromagnetic metallic state at $\mathrm{T}<\mathrm{T}_{C}$. In the region of $0.50<\mathrm{x}<0.80$, Mössbauer results indicate that the Jahn-Teller coupling becomes stronger again, which accompanied by larger change in Mn-O bond length. ${ }^{7,21}$ The larger energy separation between $d_{z^{2}}$ and $d_{x^{2}-y^{2}}$ tends to trap electrons in the low-lying energy level orbitals, and hence localize the conduction electrons. Therefore, for the samples with $\mathrm{x}>0.5$, the ground state becomes insulating and antiferromagnetic again. For the samples with $\mathrm{x}>0.90$, the local structural distortion decreases due to the lack of $\mathrm{Mn}^{3+}$ Jahn-Teller ions.

In the case of ferromagnetic materials, electric quadrupole interaction is a perturbation term in comparison with magnetic hyperfine interaction. It will become the main term at $\mathrm{T}>\mathrm{T}_{c}$. Mössbauer spectra collected at $\mathrm{T}>\mathrm{T}_{C}$ provide a possibility to determine quadrupole splitting precisely. As illustrated in fig. 3, the quadrupole splitting at $\mathrm{Fe}^{3+}$ ion is found to have no obvious change at $\mathrm{T}>\mathrm{T}_{C}$, which is same as that in $\mathrm{La}_{1-x} \mathrm{Ca}_{x} \mathrm{Mn}_{0.9575} \mathrm{Fe}_{0.0425} \mathrm{O}_{3}{ }^{13}$ These results suggest no abrupt change in the difference of $\mathrm{Mn}-\mathrm{O}$ bond lengths above $\mathrm{T}_{C}$, in agreement with the results of extended X-ray absorption fine structure (EXAFS) and neutron pair distribution function (PDF). ${ }^{6,7,22}$ Further work is needed to investigate quadrupole splitting and Jahn-Teller coupling below $\mathrm{T}_{C}$ using ${ }^{57}$ Fe-enriched samples.

In conclusion, we employed ${ }^{57} \mathrm{Fe}$ nucleus as a micro-probe to detect the local structure of $\mathrm{Mn}(\mathrm{Fe}) \mathrm{O}_{6}$ octahedra in Fe-doped $\mathrm{La}_{1-x} \mathrm{Ca}_{x} \mathrm{MnO}_{3}$ pervoskites. Our work not only confirms the local structural distortion of $(\mathrm{Mn}, \mathrm{Fe}) \mathrm{O}_{6}$ octahedron, but also reveals that Mössbauer spectroscopy can be effectively employed to estimate the Jahn-Teller coupling of these perovskites.

This work was supported by the State Key Project of Fundamental Research, the National Natural Sciences Foundation of China, as well as the Natural Sciences and Engineering 
Research Council of Canada. Z.H.C thanks the Killam Foundation for financial support. 


\section{REFERENCES}

[1] S. Jin, T. Tiefel, M. McMormack, P.A. Fastnacht, R.Ramesh and L.H. Chen, Science, 264, 413 (1994).

[2] P.Schiffer, A.P. Ramirez, W. Bao and S.W. Cheong, Phys. Rev. Lett. 75, 3336(1995).

[3] A.J. Millis, P.B. Littlewood, and B.I. Shraiman, Phys. Rev. Lett. 74,5144 (1995).

[4] H.Y.Hwang, S.W. Cheong, P.G. Radaelli, M. Marezio, and B. Batlogg, Phys. Rev. Lett. 75,914 (1995).

[5] H. Röder, J. Zang, and A.R. Bishop, Phys. Rev. Lett. 76, 1356 (1996).

[6] C.H. Booth, F.Bridges, G.H. Kwei, J.M. Lawrence, A.L. Cornelius and J.J. Neumeier, Phys. Rev. Lett. 80, 853 (1998).

[7] A.J. Millis, Nature, 392, 147(1998).

[8] J.B.A.A. Elemans, B. Van Laar, K.R. van der Veen and B.O. Loopstra, J. Solid State. Chem. 3, 238 (1971).

[9] G.H. Jonker, Physica (Amsterdam), 20, 1118 (1954).

[10] R.D. Shannon, Acta. Cryst. A32, 751 (1976).

[11] A. Simopoulous, M. Pissas, G. Kallias, E. Devlin, N. Moutis, I. Panagiotopoulous, D. Niarchos, C. Christides, and R. Sonntag, Phys. Rev. B, 59, 1263 (1999).

[12] S.B.Ogale, R.Shreekala, R.Bathe, S.K. Date, S.I. Patil, B. Hannoyer, F.Petit and G.Marest, Phys. Rev. B., 57,7841(1998).

[13] B.Hannoyer,G. Marest, J.M.Greneche, R. Bathe, S.I. Patil and S.B. Ogale, Phys. Rev. B, 61,9613(2000).

[14] A. Nath., J. Solid State Chem. 155,116(2000).

[15] U. Shimony and J.M. Knudsen, Phys. Rev. 144, 361 (1966). 
[16] K. H. Ahn, X. W. Wu, K. Liu, and C. L. Chien Phys. Rev. B 54,15299(1996)

[17] J.S. Griffith, The Theory of Transition-Metal Ion (Cambridge Univ. Press, London, 1964).

[18] S. Fraga, Handbook of Atomic Data (Elsevier Scientific Publishing Company, Amsterdam, 1976).

[19] N.N. Greewood and T.C. Gibb, Mössbauer Spectroscopy (Chapman and Hall Ltd. London, 1971).

[20] R. R. Sharma and T. P. Das, J. Chem. Phys. 41, 3581 (1964); J.O. Artman, J. O. Artman, A. H. Muir, Jr. and H. Wiedersich Phys. Rev. 173, 337(1968); R.M. Sternheimer, Phys.Rev. 130, 1423 (1963)

[21] P.G. Radaelli, D.E. Cox, M. Marezio, S.W. Cheong, Phys. Rev. B. 55,3015(1997).

[22] S.J.L. Billinge, R.G. DiFrancesco, G.H. Kwei, J.J. Neumeier and J.D. Thompson, Phys. Rev. Lett. 77,853(1996) 


\section{Figure captions}

Figure 1. Room temperature ${ }^{57} \mathrm{Fe}$ Mössbauer spectra of $\mathrm{La}_{0.69} \mathrm{Ca}_{0.31} \mathrm{Mn}_{0.91} \mathrm{Fe}_{0.09} \mathrm{O}_{3}$ (a) and $\mathrm{La}_{0.69} \mathrm{Ca}_{0.31} \mathrm{Mn}_{0.96} \mathrm{Fe}_{0.04} \mathrm{O}_{3}(\mathrm{~b})$.

Fig. 2. Quadrupole splitting at $\mathrm{Fe}^{3+}$ ion and Jahn-Teller coupling in $\mathrm{La}_{1-x} \mathrm{Ca}_{x} \mathrm{Mn}_{0.91} \mathrm{Fe}_{0.09} \mathrm{O}_{3} \quad(\mathrm{x}=0.00-1.00)$ and $\mathrm{La}_{1-x} \mathrm{Ca}_{x} \mathrm{Mn}_{0.96} \mathrm{Fe}_{0.04} \mathrm{O}_{3} \quad(\mathrm{x}=0.31-0.60) \quad$ perovskites at room temperature.

Fig. 3. Temperature dependence of quadrupole splitting at $\mathrm{Fe}^{3+}$ ion and Jahn-Teller coupling in $\mathrm{La}_{1-x} \mathrm{Ca}_{x} \mathrm{Mn}_{0.91} \mathrm{Fe}_{0.09} \mathrm{O}_{3}$ and $\mathrm{La}_{1-x} \mathrm{Ca}_{x} \mathrm{Mn}_{0.96} \mathrm{Fe}_{0.04} \mathrm{O}_{3}$ ( $\mathrm{x}=0.31$ and 0.50) 
Relative transmission Relative transmission

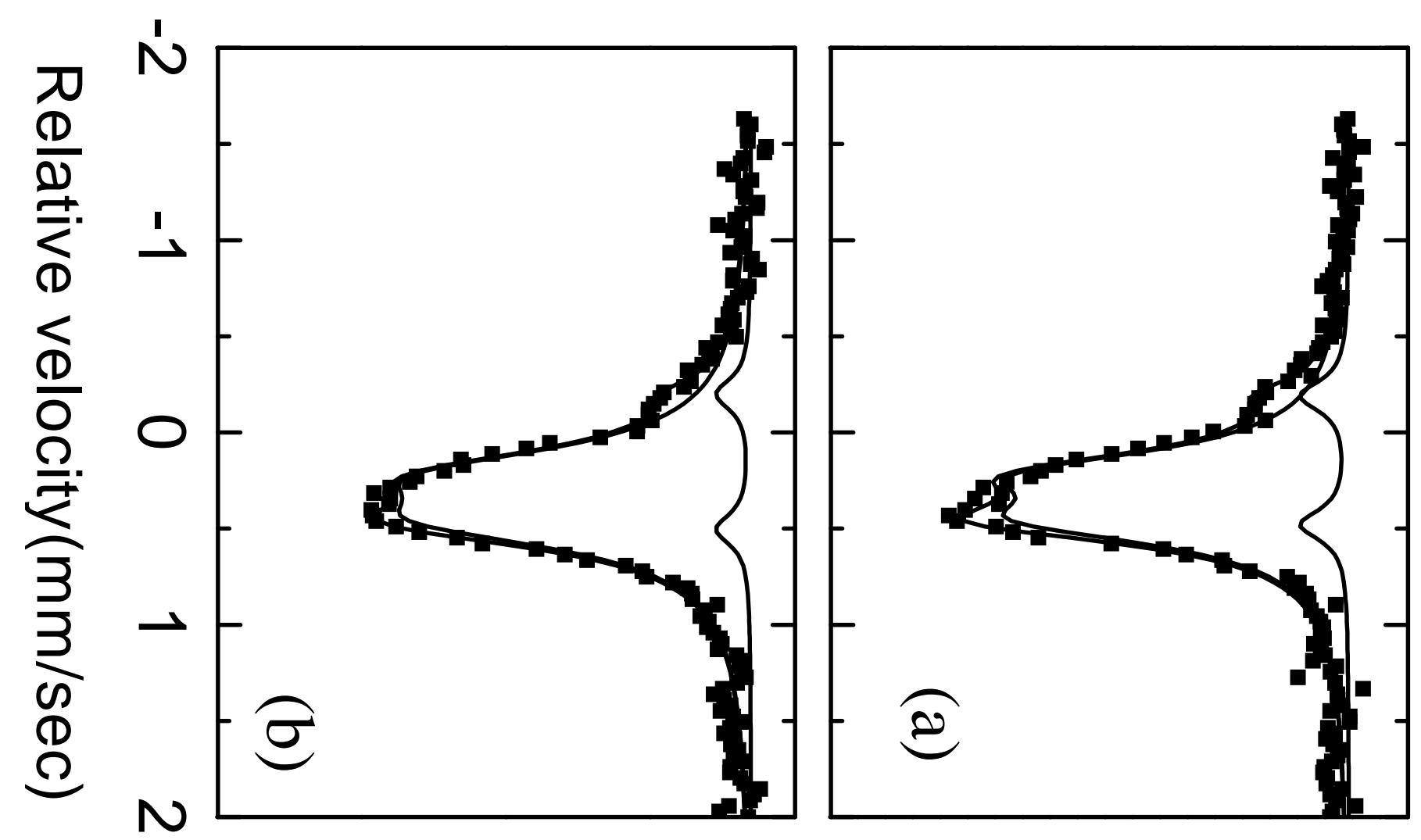




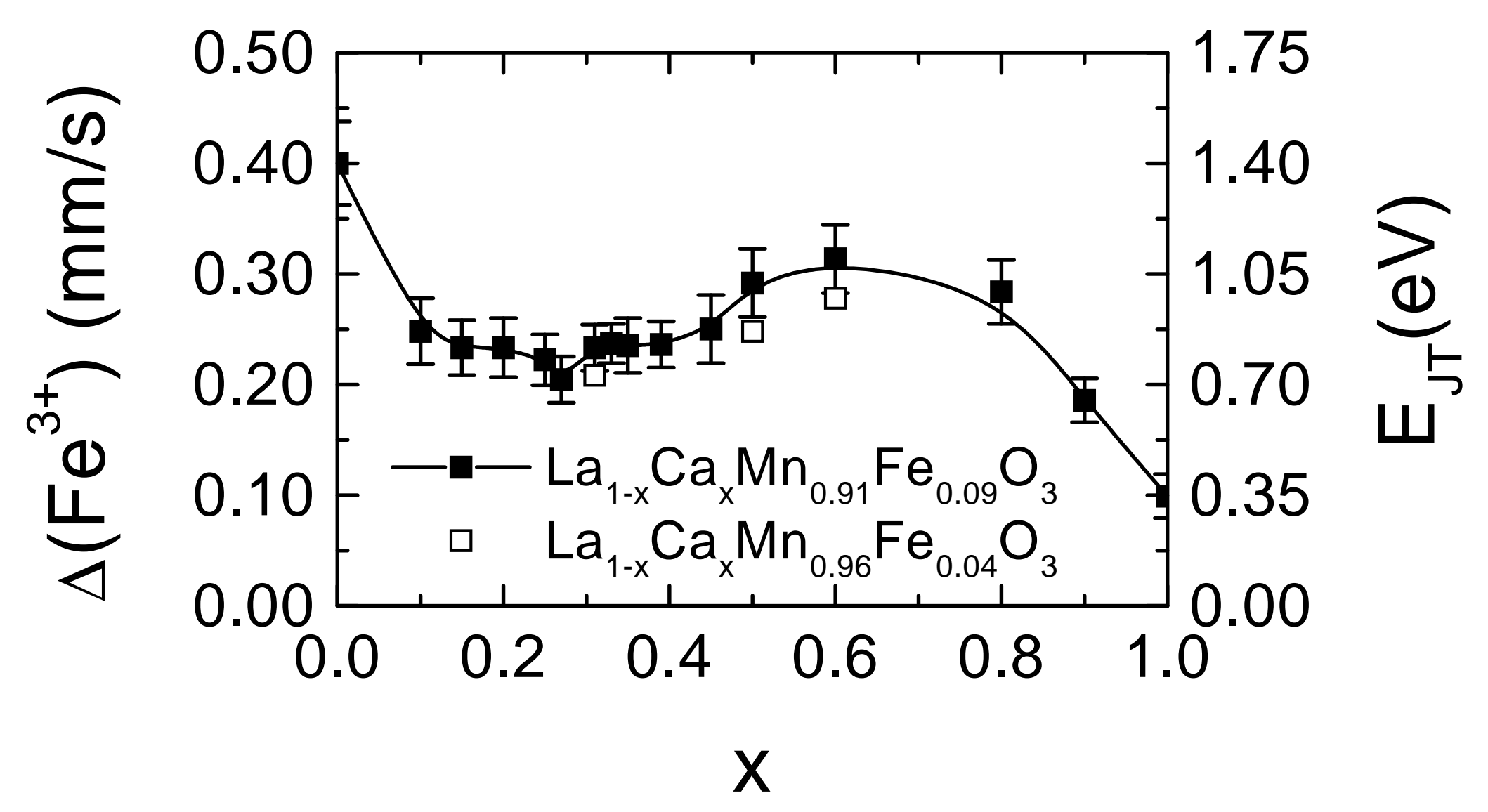




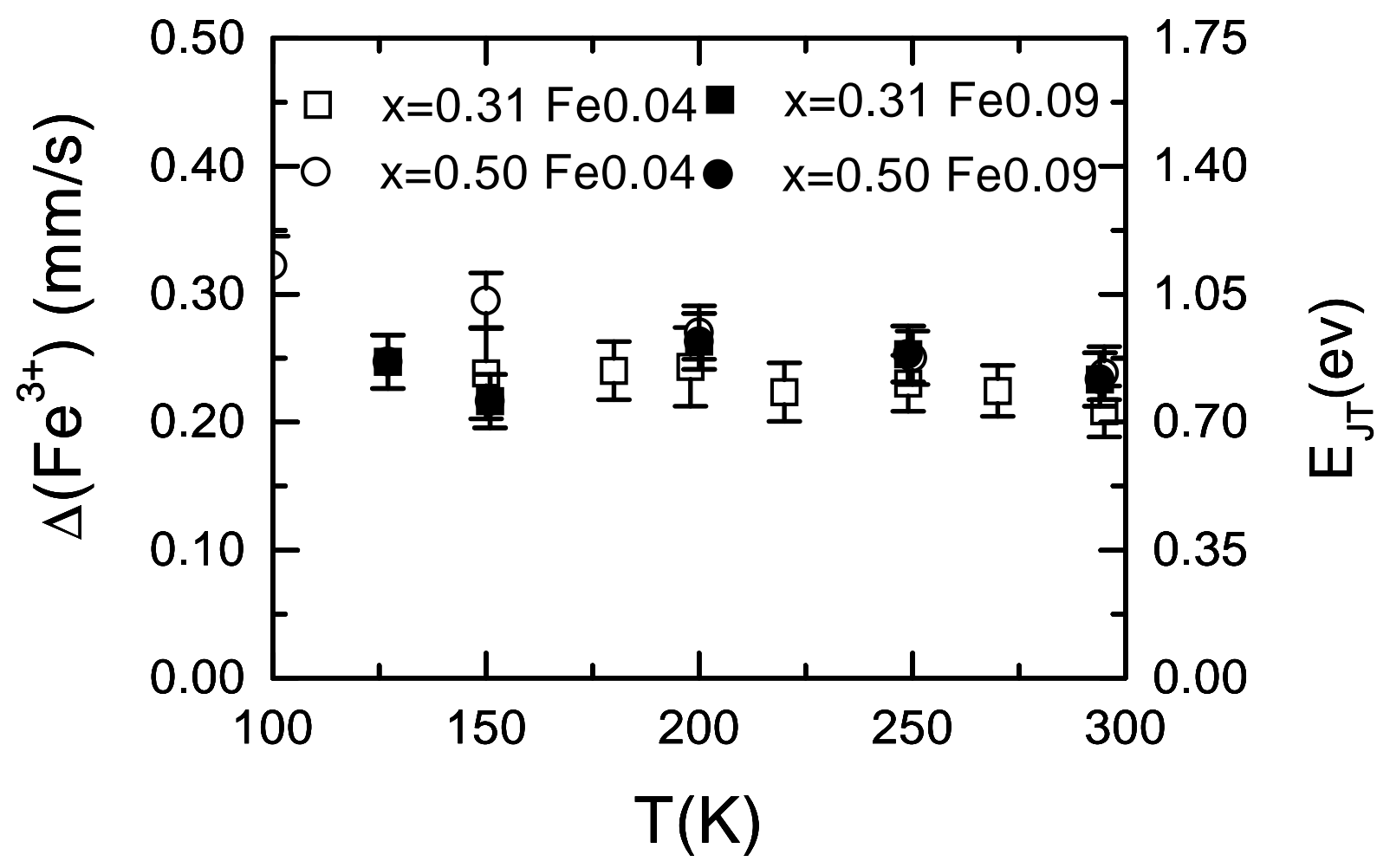

